\title{
An Investigation of Grounding Resistance Estimation of Human Body by Attenuation Coefficient
}

\author{
Jeu-Min Lin \\ Department of Electrical Engineering, National Cheng Kung University, Taiwan
}

Copyright $\mathrm{C} 2017$ by authors, all rights reserved. Authors agree that this article remains permanently open access under the terms of the Creative Commons Attribution License 4.0 International License

\begin{abstract}
It is dangerous when human are working in and around the high voltage environments. The safety of personnel in and around electric power the installations has been a prime concern. Specifically, this safety is generally in terms of the allowable touch and step voltages. These voltages are related to the ground resistance of human foot. In order to increase the safety of personnel, a high resistivity surface layer of gravel is normally existed in the substation switchyards or other high voltage areas. The thickness of this layer will affect the calculation of the ground resistance of human foot. Among them, the electromagnetic field factors are also considered in this paper. As a result, the surface layer of gravel can be used to reduce the ground resistance of human foot. The equivalent model of human foot was modeled by the circular plate conductor in the past. This paper investigates the present methods for estimating the ground resistance of human foot and proposes an equivalent model for calculating the ground resistance of human foot. A comparison of the proposed model and present methods is also shown in this paper.
\end{abstract}

Keywords Attenuation Coefficient, Step Voltage, Contact Voltage, Body Grounding Resistance, Electromagnetic Field Factor

\section{Introduction}

In the high voltage environments, such as work in the substations, high voltage electric towers side, railways, or Underground Railroad track sides, the ground surface is usually evenly covered with a layer of 8 to $25 \mathrm{~cm}$ thickness of gravel, to increase the soil and the contact resistance between the feet (Contact Resistance). Assuming that the equivalent resistance of the shoes and socks is zero, the contact resistance is the equivalent resistance of the gravel layer. Because the contact resistance between the soil layer and the foot is large, when the accident occurs (for example, the hand touches the lightning striking device), the current flowing through the body will be much smaller than the absence of the gravel layer, in other words, The contact voltage or step voltage suffered by the human body will be smaller. In this way, we can ensure the safety of the site workers.

In the calculation of the human body can withstand the contact voltage or step voltage, the foot of the ground resistance is a very important factor. In general, the human foot is often equivalent to a circular plate Conductor with a radius of $8 \mathrm{~cm}$. According to the IEEE standard 80 , a circular plate-like conductor having a radius $b$ is placed on a soil having a resistivity $\rho_{\mathrm{s}}$ (assuming a fairly uniform distribution of the resistivity distribution), and the ground resistance $R_{f}$ thereof can be expressed as follows [1]:

$$
\mathrm{R}_{\mathrm{f}}=\frac{\rho_{\mathrm{s}}}{4 \mathrm{~b}}
$$

Where $\mathrm{b}=0.08 \mathrm{~m}$

$$
\mathrm{R}_{\mathrm{f}} \approx 3 \rho_{\mathrm{s}}
$$

Since the size of the gravel layer is large enough, it is possible to assume that the resistivity of the material in the foot is uniform in the horizontal direction. However, compared with the equivalent radius of the foot $(0.08 \mathrm{~m})$, the thickness of the gravel layer is not large enough, so the resistivity of the material to which the foot is exposed is not uniform in the vertical direction. If the high resistivity gravel is laid on a low resistivity soil, this will cause the ground resistance of the foot to be lower than equation (1). At this time, the ground resistance of the foot can be expressed as follows [2]:

$$
\mathrm{R}_{\mathrm{f}}=\frac{\rho_{\mathrm{c}}}{4 \mathrm{~b}} \mathrm{C}
$$

Where $\rho_{c}=$ the resistivity of the gravel layer

$\mathrm{C}=$ attenuation coefficient

The paper should note that if you consider the gravel layer, in the ground resistance, the IEEE standard 80 has derived the grounding resistance of the infinite series 
expression (Infinite Series Expression). However, in the reference [8], this infinite series is deduced into a finite expression. The ground resistors calculated by the two representations are quite close, but the finite term expressions are more convenient to use. The structure of this paper is as follows: The method of calculating the grounding resistance of the human body foot will be described in Section 2, and then we will briefly introduce the method mentioned in this paper and the simulation results listed in Section IV. Finally, it is the conclusion of this paper

\section{The Existing Calculation of the Human Body Foot Grounding Resistance Method}

According to the IEEE standard 80 , the foot is equivalent to a circular plate-shaped conductor with a radius $b$ and placed on a double-layer medium. The first layer is a gravel of resistivity $\rho_{\mathrm{c}}$, the second layer is a soil with resistivity $\rho_{\mathrm{s}}$, the ground resistance $R_{f}$ of the foot can be expressed as follows [1]:

$$
\begin{gathered}
\mathrm{R}_{\mathrm{f}}=\frac{\rho_{\mathrm{c}}}{4 \mathrm{~b}} \mathrm{~F}(\mathrm{x}) \\
\mathrm{F}(\mathrm{x})=1+2 \sum_{\mathrm{n}=1}^{\infty} \frac{\mathrm{K}^{\mathrm{n}}}{\sqrt{1+(2 \mathrm{nx})^{2}}} \\
\mathrm{~K}=\frac{\rho_{\mathrm{s}}-\rho_{\mathrm{c}}}{\rho_{\mathrm{s}}+\rho_{\mathrm{c}}}
\end{gathered}
$$

Where $\rho_{c}=$ the resistivity of the gravel layer

$\mathrm{P}_{\mathrm{S}}=$ resistivity of soil layer

$\mathrm{X}=\mathrm{h} / \mathrm{b}$

$\mathrm{H}=$ thickness of gravel layer

$\mathrm{B}=$ equivalent radius of the foot $(0.08 \mathrm{~m})$

$\mathrm{K}=$ Reflection Factor

According to the hemispherical electrode (Hemispherical Electrode) resistance method, and the use of virtual image method (Method of Image) [2], can be derived equations (4), (5) and (6). In order to simplify the complex mathematical operations, so in the derivation of the process made some assumptions, but this has caused the equation produced an error. Especially when $\mathrm{h}$ smaller, the error will be greater [5]. When $\mathrm{K}$ is equal to $-0.2,-0.4,-0.6$, -0.8 , the relationship between $\mathrm{F}(\mathrm{x})$ and $\mathrm{h}$ is shown in Fig. 1 . As can be seen from the figure, the slope of the curve increases as $\mathrm{h}$ decreases, but when $\mathrm{h}$ is small, the slope of the curve is reduced.

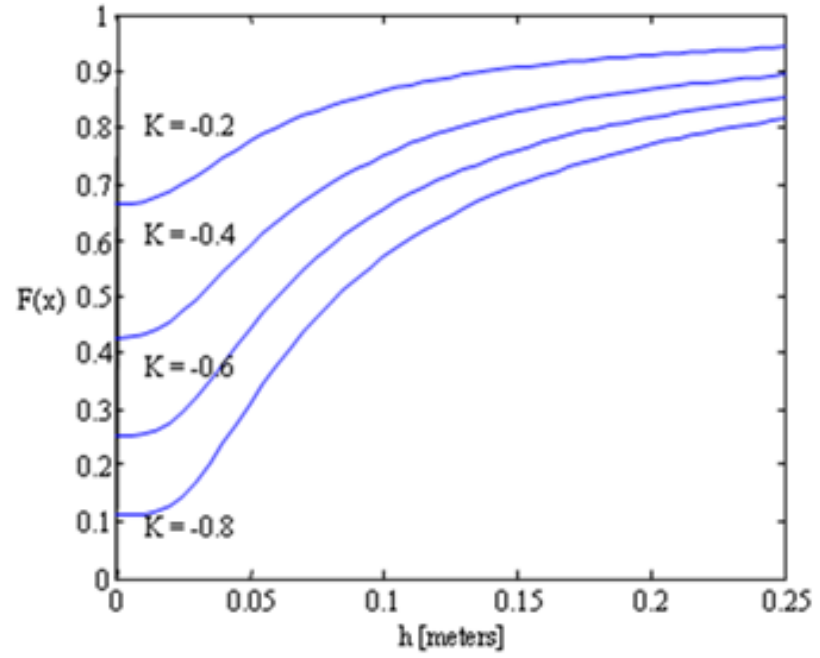

Figure 1. $\mathrm{F}(\mathrm{x})$ and $\mathrm{h}, \mathrm{K}$ relationship between the maps

In another method of grounding resistance, the equivalent model of the foot is also a circular plate conductor, but the derivation method is different from the derivation method of the IEEE standard 80 equation.

If the foot is equivalent to a radius of $b(=0.08 \mathrm{~m})$ of the circular plate-like conductor, placed in the resistivity $\rho$ s on the soil (assuming a fairly uniform distribution of resistivity), the grounding resistance can be expressed as follows [1]:

$$
\mathrm{R}_{\mathrm{f}}=\frac{\rho_{\mathrm{s}}}{4 \mathrm{~b}}
$$

If the gravel layer is considered, the ground resistance can be expressed as follows $[3,4,5]$ :

$$
\begin{gathered}
\mathrm{R}_{\mathrm{f}}=\frac{\rho_{\mathrm{c}}}{4 \mathrm{~b}} \mathrm{H}(\mathrm{x}) \\
\mathrm{H}(\mathrm{x})=1+\frac{4}{\pi} \sum_{\mathrm{n}=1}^{\infty} \mathrm{P}
\end{gathered}
$$

$$
\begin{gathered}
\mathrm{P} \approx \frac{\mathrm{K}^{\mathrm{n}}}{2 \mathrm{nx}}\left[1-\frac{7}{12} \frac{1}{(2 \mathrm{nx})^{2}}+\frac{33}{40} \frac{1}{(2 \mathrm{nx})^{4}}\right] \\
\mathrm{K}=\frac{\rho_{\mathrm{s}}-\rho_{\mathrm{c}}}{\rho_{\mathrm{s}}+\rho_{\mathrm{c}}}
\end{gathered}
$$

When $(2 \mathrm{~h}) / \mathrm{b}>3.5$ or $\mathrm{h}>0.14 \mathrm{~m}$, the values obtained by Equations (8) and (9) are more accurate. Please refer to Appendix $\mathrm{A}$ for restrictions on this scope. When $\mathrm{K}$ is equal to $-0.2,-0.4,-0.6,-0.8$, the relationship between $\mathrm{H}(\mathrm{x})$ and $\mathrm{h}$ is shown in Fig. 2. 


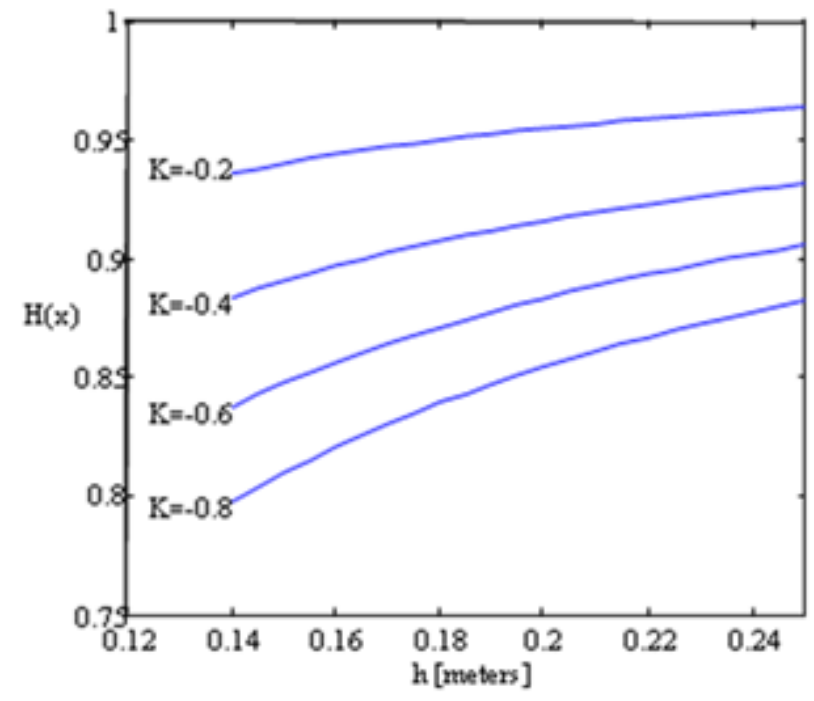

Figure 2. The map of the relationship of $\mathrm{H}(\mathrm{x})$ and $\mathrm{h}, \mathrm{K}$

Then, the design can be synthesized with ease that transfers the descriptions to gate-level net lists without further effort.

\section{The Proposed Method}

As shown in Fig. 3, a plate-like conductor is placed on a double-layer medium, the first layer is a gravel of resistivity $\rho c$, and the second layer is a soil with resistivity $\rho_{\mathrm{s}}$. Among them, the gravel layer thickness $\mathrm{h}$. We can use the virtual image method we can get plate-like conductor and its mirror (Images) position distribution. Assuming that the ground current of the plate conductor is $\mathrm{I}$, if the boundary between the air and the gravel layer is the mapping interface, the Current is not the same; if the boundary between the soil layer and the gravel layer As the mapping interface, then the current will be proportional to the power of the reflection factor $\mathrm{K}$, as shown in Fig. 4. Where $K=(\rho s-\rho c) /\left(\rho_{s}+\rho_{c}\right)$. The resistivity of all the regions in Fig. 4 is $\rho_{c}[10]$, whether the plate conductor itself or its mirror is discharged to the gravel layer with resistivity $\rho_{\mathrm{c}}$.

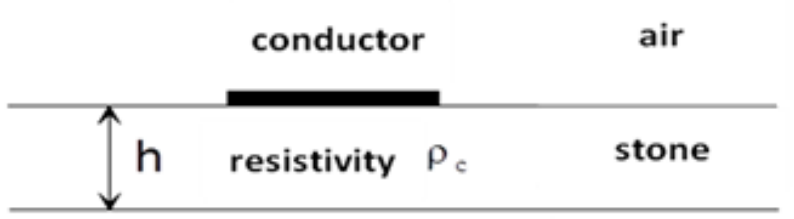

soil

Figure 3. Schematic representation of a square plate conductor in a gravel layer

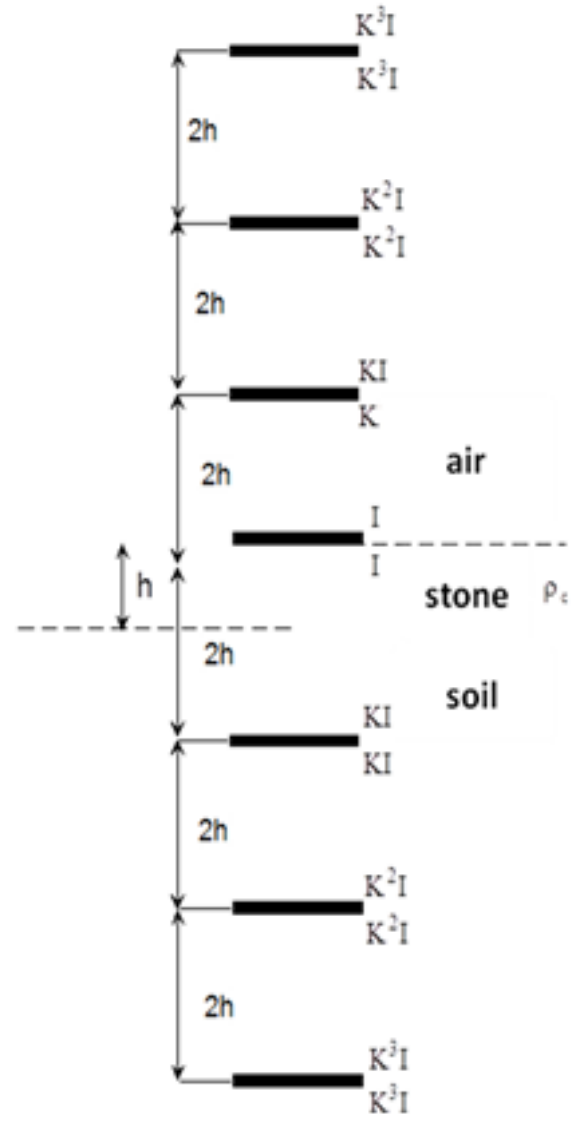

Figure 4. Square plate-like mapping architecture

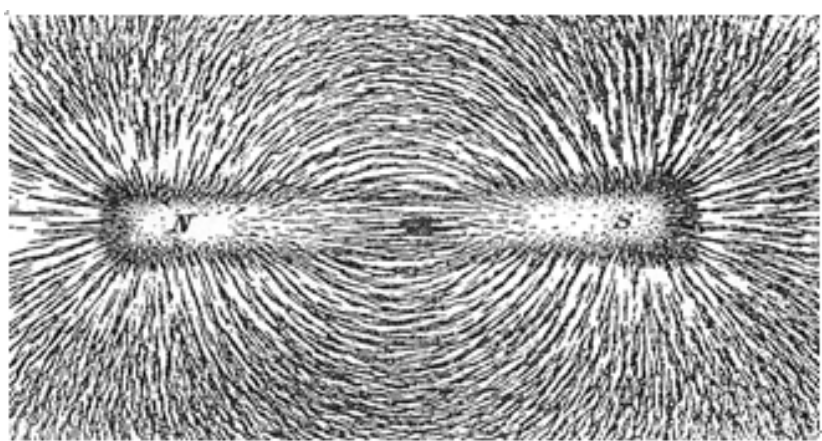

Figure 5. The diagram of magnetic field

Using the superposition principle (Superposition Theorem), the average potential $\mathrm{V}_{\text {ave }}$ of the plate-like conductor can be obtained as follows, which also consider the factors of electromagnetic field in the proposed paper. Fig. 5 shows a schematic view of the magnetic field lines displayed through the iron powder on the white paper, which is known that the pure quantity of the electromagnetic field function has an important influencing factor on the creatures, so that [11]: 


$$
\mathrm{V}_{\mathrm{p}}=2 \pi \mathrm{r} \sigma\left[\sinh ^{-1} \frac{\beta \ell}{\mathrm{d}}+\sinh ^{-1} \frac{(1-\beta) \ell}{\mathrm{d}}\right]
$$

Where $r=$ the radius of the wire, $\sigma=$ surface charge density.

$$
\begin{gathered}
\mathrm{V}_{\mathrm{ave}}=2 \mathrm{IR}+4 \mathrm{KIR}_{\mathrm{m}(2 \mathrm{~h})}+4 \mathrm{~K}^{2} \mathrm{IR}_{\mathrm{m}(4 \mathrm{~h})}+\cdots \\
=I\left[2 R+4 \sum_{n=1}^{\infty} K^{n} R_{m(2 n h)}\right]+\rho \\
\boldsymbol{\rho}=\nabla \cdot \mathbf{D} \\
R_{p}=\frac{V_{\text {ave }}}{I}=2 R+4 \sum_{n=1}^{\infty} K^{n} R_{m(2 n h)}
\end{gathered}
$$

Where $\mathrm{R}=$ plate-like conductor grounding resistance obtained in a uniform medium of resistivity $\rho_{c}$

$\mathrm{R}_{\mathrm{m}}\left(2_{\mathrm{nh}}\right)=$ mutual resistance of two parallel plate-like conductors spaced $2_{\text {nh }}$ apart in a uniform medium of resistivity $\rho_{\mathrm{c}}$ (Mutual Ground Resistance)

$\boldsymbol{\rho}=$ Volume charge density

$\nabla=$ gradient operator

$\mathbf{D}=$ electric flux density

$$
\text { . }=\text { dot operator }
$$

Assuming that the length of the strip conductor is $\ell$, the width is a and the thickness is $b$, the ground resistance $R$ of the strip conductor in the uniform medium of the resistivity $\rho_{c}$ is as follows [10]:

$$
\mathrm{R}=\frac{\rho_{\mathrm{c}}}{2 \pi \ell}\left[\ln \frac{2 \ell}{\mathrm{a}}+\frac{\mathrm{a}^{2}-\pi \mathrm{ab}}{2(\mathrm{a}+\mathrm{b})^{2}}\right]
$$

When the width $a$ is much larger than the thickness $b$ and the length is equal to the width a, the ribbon conductor can be regarded as a square plate conductor,

$$
\begin{gathered}
R \approx 0.5966 \frac{\rho_{c}}{\pi \ell} \\
\mathrm{V}_{\mathrm{p}}=2 \pi \mathrm{r} \sigma\left[\sinh ^{-1} \frac{\beta \ell}{\mathrm{d}}+\sinh ^{-1} \frac{(1-\beta) \ell}{\mathrm{d}}\right]
\end{gathered}
$$

Where $r=$ radius of the wire,

$\Sigma=$ surface charge density

As shown in Fig. 6, the two metal lines are long and spaced apart from each other. If the surface charge distribution of the metal line $\mathrm{AB}$ is uniform, the potential $\mathrm{Vp}$ at any point on the wire $\mathrm{CD}$ can be expressed as

$$
\mathrm{V}_{\mathrm{p}}=2 \pi \mathrm{r} \sigma\left[\sinh ^{-1} \frac{\beta \ell}{\mathrm{d}}+\sinh ^{-1} \frac{(1-\beta) \ell}{\mathrm{d}}\right]
$$

Where $r=$ the radius of the wire, $\sigma=$ surface charge density.

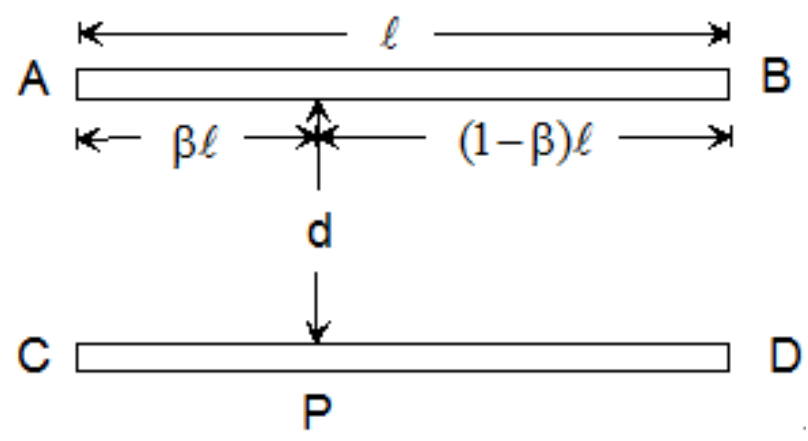

Figure 6. The geometric position on the two parallel lines

The average potential $\mathrm{V}_{\text {ave }}$ of the metal wire $\mathrm{CD}$ is:

$$
\begin{aligned}
\mathrm{V}_{\text {ave }} & =2 \pi \mathrm{r} \sigma \int_{0}^{1}\left[\sinh ^{-1} \frac{\beta \ell}{\mathrm{d}}+\sinh ^{-1} \frac{(1-\beta) \ell}{\mathrm{d}}\right] \mathrm{d} \beta \\
& =4 \pi \mathrm{r} \sigma\left(\sinh ^{-1} \frac{\ell}{\mathrm{d}}-\sqrt{1+\frac{\mathrm{d}^{2}}{\ell^{2}}}+\frac{\mathrm{d}}{\ell}\right) \\
& =\frac{2 \mathrm{Q}}{\ell}\left(\sinh ^{-1} \frac{\ell}{\mathrm{d}}-\sqrt{1+\frac{\mathrm{d}^{2}}{\ell^{2}}}+\frac{\mathrm{d}}{\ell}\right)
\end{aligned}
$$

Where $\mathrm{Q}=$ total charge of metal line $\mathrm{AB}$.

Since the square plate-like conductor can be visually composed of an infinite number of metal wires, the average potential caused by a charged square-shaped conductor on the other square-shaped conductor is also equation (17). So the mutual resistance $\mathrm{R}_{\mathrm{m}(\mathrm{d})}$ can be expressed as [9]

$$
\begin{array}{r}
\mathrm{R}_{\mathrm{m}(\mathrm{d})}=\frac{\rho_{\mathrm{c}}}{4 \pi \mathrm{C}}=\frac{\rho_{\mathrm{c}}}{4 \pi} \frac{\mathrm{V}_{\mathrm{ave}}}{\mathrm{Q}} \\
=\frac{\rho_{c}}{2 \pi \ell}\left(\sinh ^{-1} \frac{\ell}{d}-\sqrt{1+\frac{d^{2}}{\ell^{2}}}+\frac{d}{\ell}\right)
\end{array}
$$

Where $\rho_{\mathrm{c}}=$ the resistivity of the environment in which the conductor is located.

Therefore, the resistance of the two-sided plate-shaped conductor at a distance of $2 \mathrm{nh}$ is

$$
\begin{aligned}
\mathrm{R}_{\mathrm{m}(2 \mathrm{nh})} & =\frac{\rho_{\mathrm{c}}}{2 \pi \ell}\left(\sinh ^{-1} \frac{\ell}{2 \mathrm{nh}}-\sqrt{1+\frac{(2 \mathrm{nh})^{2}}{\ell^{2}}}\right. \\
& \left.+\frac{2 \mathrm{nh}}{\ell}\right)
\end{aligned}
$$

Equations (15) and (22) are substituted into Equation (13), so that the ground resistance $R_{f}$ after the equivalent of a square plate-like conductor can be obtained as follows:

$$
\begin{aligned}
& \mathrm{R}_{\mathrm{f}}=1.1931 \frac{\rho_{\mathrm{c}}}{\pi \ell} \mathrm{S}(\mathrm{y}) \\
& \mathrm{S}(\mathrm{y})=1+1.6762 \sum_{\mathrm{n}=1}^{\infty} \mathrm{T}
\end{aligned}
$$




$$
\begin{gathered}
\mathrm{T}=\mathrm{K}^{\mathrm{n}}\left[\sinh ^{-1} \frac{1}{2 \text { ny }}+2 \mathrm{ny}-\sqrt{(2 \mathrm{ny})^{2}+1}\right] \\
\mathrm{K}=\left(\rho_{\mathrm{s}}-\rho_{\mathrm{c}}\right) /\left(\rho_{\mathrm{s}}+\rho_{\mathrm{c}}\right)
\end{gathered}
$$

Where $\rho_{\mathrm{c}}=$ the resistivity of the gravel layer,

$\rho_{\mathrm{s}}=$ resistivity of soil layer,

$\mathrm{h}=$ thickness of gravel layer,

$\ell=$ Side length of square plate conductor,

$\mathrm{y}=\mathrm{h} /$,

$\mathrm{K}=$ reflection factor

By the way in this paper, when $(2 \mathrm{~h}) />1$, the values obtained by equations (23) and (24) are more accurate.

\section{Actual Field Testing}

If the coefficient ( $\rho c / 4 b$ ) of equation (23) is equal to the coefficient of equation $(24)(1.1931 \rho \mathrm{c} / \pi),=0.1215 \mathrm{~m}$. In this way, when $\mathrm{h}>0.06 \mathrm{~m}$, the value obtained by equation (21) is more accurate. Therefore, when $\mathrm{K}$ is equal to -0.2 , $-0.4,-0.6,-0.8$, the relationship between $\mathrm{S}(\mathrm{y})$ and $\mathrm{h}$ is shown in Fig. 7. If $\rho s=100[\Omega \cdot \mathrm{m}], \mathrm{K}$ is equal to $0,-0.1$, $-0.2, \ldots,-0.9$, the relationship between $\mathrm{R}_{\mathrm{f}}$ and $\mathrm{h}$ is shown in Fig. 7. We can see from Fig. 7 the following described the phenomenon. When the thickness of the gravel layer remains constant, the ground resistance of the foot decreases as the reflection factor increases. When the reflection factor remains constant, the ground resistance of the foot decreases as the thickness of the gravel layer decreases. Table 1 shows the comparison of the respective attenuation factors. To observe these data, we find that $\mathrm{S}(\mathrm{y})$ is quite close to $\mathrm{H}(\mathrm{x})$ and in any case $\mathrm{F}(\mathrm{x})$ is less than $\mathrm{H}(\mathrm{x})$ and $S(y)$. In addition, when $K=0$, regardless of $h$ for several, the attenuation factor is 1 . Since $K=0$ means that the resistivity of the soil layer is the same as that of the gravel layer. Therefore, regardless of any of the grounding resistance formula in this paper, the value obtained is equal with. On the other hand, we can also find that the effect of $\mathrm{K}=0$ is exactly the same as $\mathrm{h}=0$ and the resistivity of the soil layer is equal to the resistivity of the gravel layer.

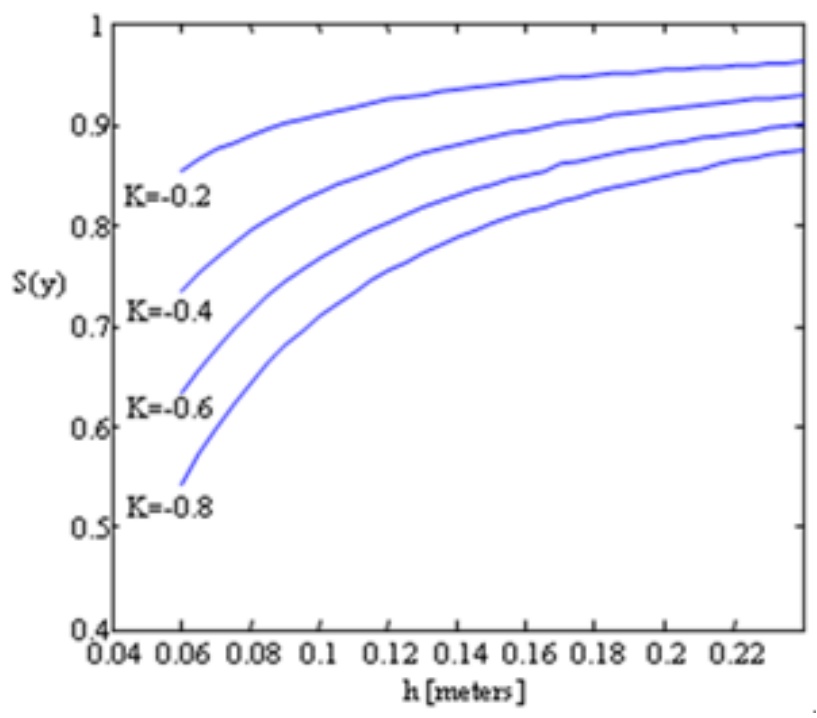

Figure 7. The map of the relationships of $\mathrm{S}(\mathrm{y}), \mathrm{h}$ and $\mathrm{K}$

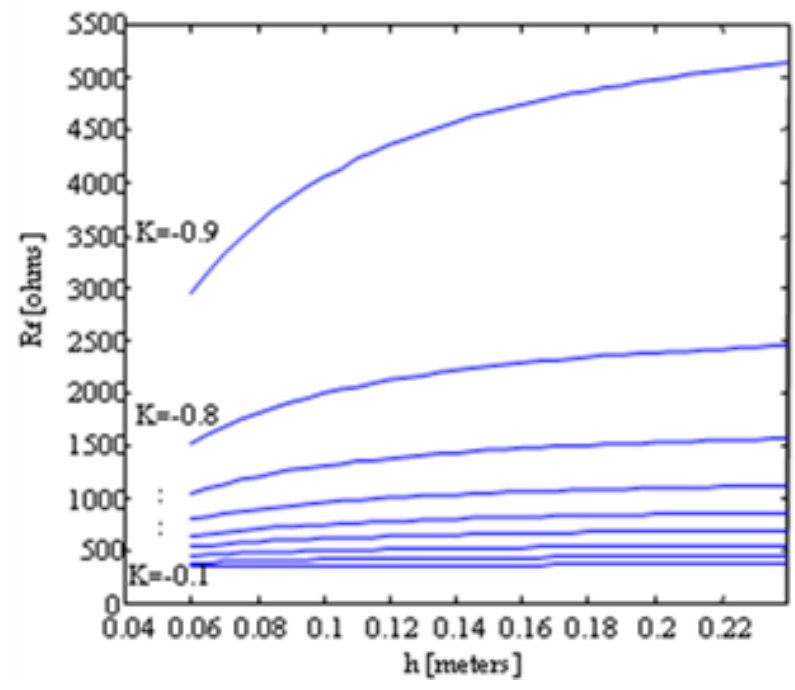

Figure 8. The map of the relationships of $\mathrm{Rf}$ and $\mathrm{h}, \mathrm{K}$ relation 
Table 1. A comparison table of attenuation coefficient

\begin{tabular}{|c|c|c|c|c|}
\hline $\mathrm{K}$ & $\mathrm{h}(\mathrm{cm})$ & $\mathrm{F}(\mathrm{x})$ & $\mathrm{H}(\mathrm{x})$ & - \\
\hline 0.0 & 6 & 1 & - & 1 \\
0.0 & 10 & 1 & 1 & 1 \\
0.0 & 14 & 1 & - & 0.9247 \\
0.0 & 20 & 1 & - & 0.9529 \\
\hline-0.1 & 10 & 0.8950 & 0.9668 & 0.9659 \\
-0.1 & 14 & 0.9294 & 0.9763 & 0.9759 \\
\hline-0.1 & 20 & 0.9477 & - & 0.7937 \\
-0.1 & 6 & 0.9626 & - & 0.8705 \\
-0.3 & 10 & 0.7145 & 0.9061 \\
-0.3 & 14 & 0.8066 & 0.9090 & 0.9337 \\
-0.3 & 20 & 0.8564 & - & 0.6824 \\
-0.3 & 6 & 0.8973 & - & 0.8002 \\
-0.5 & 10 & 0.5639 & 0.8597 & 0.8551 \\
-0.5 & 14 & 0.7025 & 0.8994 & 0.8976 \\
-0.5 & 20 & 0.7787 & - & 0.5860 \\
\hline-0.5 & 6 & 0.8415 & - & 0.7389 \\
-0.7 & 10 & 0.4353 & 0.8168 & 0.8105 \\
-0.7 & 14 & 0.6124 & - & 0.8661 \\
\hline-0.9 & 20 & 0.7110 & - & 0.5009 \\
-0.9 & 6 & 0.7928 & 0.7789 & 0.6846 \\
-0.9 & 10 & 0.3237 & 0.8411 & 0.7709 \\
\hline
\end{tabular}

Remarks: $\quad \mathrm{F}(\mathrm{x})$ is the attenuation factor of IEEE standard 80

$\mathrm{H}(\mathrm{x})$ is the attenuation factor of the circular plate conductor.

$\mathrm{S}(\mathrm{y})$ is the attenuation factor of the square plate conductor.

\section{Conclusions}

Through the discussion of this article, the proposed paper summarizes the following conclusions as a reference to the design of the grounding system:

(1) If the surface covered with a layer of high resistivity gravel, can increase the contact resistance between the soil and feet. Because the contact between the soil layer and the foot is very large, so when the accident occurs, the current through the body than the absence of gravel layer is much smaller. Therefore, covered with gravel can improve the safety of the site staff.

(2) When the reflection factor remains constant, the attenuation coefficient decreases as the thickness of the gravel layer decreases. On the other hand, the contact voltage or step voltage is proportional to the ground resistance, so the thickness of the gravel layer is thinner, the human body will suffer the step voltage or contact voltage will be smaller. See Appendix B for details.

(3) When the thickness of the gravel layer remains constant, the attenuation coefficient increases as the reflection factor increases. So the greater the reflection factor, the human body suffered by the step voltage or contact voltage will be smaller. See Appendix C. for details.

(4) The attenuation coefficient $\mathrm{S}(\mathrm{y})$ and $\mathrm{H}$ (x) are quite close to each other, the error is less than $1.2 \%$. Therefore, the formulas presented in this paper are quite accurate compared with those in the references.
And the use range of S (y) (the thickness of the gravel layer) is larger than that of $\mathrm{H}(\mathrm{x})$.

(5) (Y) / F ( $\mathrm{x}$ ) in any case, the attenuation coefficient $\mathrm{F}(\mathrm{x})$ is less than $\mathrm{S}(\mathrm{y})$ and $\mathrm{H}(\mathrm{x})$, especially when $\mathrm{K}<-0.9$ and $\mathrm{h}<10 \mathrm{~cm}$, The In addition, due to the inaccuracy of the equation in IEEE Standard 80, the ground resistance standard set by it is somewhat conservative compared to other literature and this text.

(6) With the discussions of the proposed paper, this content can enhance and ensure the personal safety.

(7) Based on the Kirchhoff's law, if the resistance remains constant, the greater the voltage, the greater the current, making the larger the electromagnetic field. In other words, the phenomenon will affect the health of human being.

(8) According to the medical literatures, as time changes, high electromagnetic fields can affect the responses of human physiology and psychology.

(9) According to Coulomb's Law, it is known that the interaction between the two stationary point charges in vacuum is inversely proportional to the square of the distance. In other words, the impact of overhead lines or substations is almost zero.

(10) Known wire has resistance and inductance and capacitance, the impedance is the sum of the resistance and reactance in the vector, the resistance can be ignored at low current, and the inductance can be ignored at low frequencies. And at high current or high frequencies, these characteristics will be affected. 


\section{Appendix A}

A circular plate-like conductor having the same radius at both radii is located on the same axis and the two conductors are parallel to each other but not on the same plane. The average potential of one of the charged conductors on the other conductor is

$$
V_{\text {ave }, p}=\frac{Q}{s}\left(1-\frac{7}{12} \frac{a^{2}}{s^{2}}+\frac{33}{40} \frac{a^{4}}{s^{4}}-\cdots\right) \Rightarrow \frac{a V_{a v e, p}}{Q}=\frac{a}{s}\left(1-\frac{7}{12} \frac{a^{2}}{s^{2}}+\frac{33}{40} \frac{a^{4}}{s^{4}}-\cdots\right)
$$

Where, $a$ : The radius of a circular plate conductor,

$s$ : The distance between the centers of the two conductors,

$Q$ : The total charge of the charged conductor

Are in the same plane and the other conditions are the same, then the average potential of one of the charged conductors on the other conductor is

$$
V_{\text {ave }, \mathrm{s}}=\frac{Q}{s}\left(1+\frac{7}{24} \frac{a^{2}}{s^{2}}+\frac{99}{320} \frac{a^{4}}{s^{4}}+\cdots\right) \Rightarrow \frac{a V_{a v e, s}}{Q}=\frac{a}{s}\left(1+\frac{7}{24} \frac{a^{2}}{s^{2}}+\frac{99}{320} \frac{a^{4}}{s^{4}}+\cdots\right)
$$

When $\mathrm{s} / \mathrm{a}>3.5$, (A.1) and (A.2) in the $\mathrm{s} / \mathrm{a}>3.5$ will approach a similar value, so

$$
\begin{gathered}
\frac{V_{a v e, p}}{Q}=\frac{V_{a v e, s}}{Q} \\
\text { And } R=\frac{\rho}{4 \pi} \frac{V}{Q}
\end{gathered}
$$

Therefore, $R_{p}=R_{s}$. Where $R_{p}=$ the mutual resistance at different planes, $R_{s}=$ the mutual resistance at the same plane [10]. Therefore, when the authors choose (2h) / b> 3.5 , we will improve the accuracy of equations (8) and (9). Similarly, if $(2 \mathrm{~h}) />1$ is chosen, the values obtained from equations (20) and (21) will be more accurate.

\section{Appendix B}

The equation (23) can be obtained $\rho_{\mathrm{c}}$ by

$$
\rho_{c}=\frac{1-K}{1+K} \rho_{s}
$$

Equation (20) can be therefore rewritten as

$$
\mathrm{R}_{\mathrm{f}}=\frac{1.1931}{\pi \ell} \frac{1-\mathrm{K}}{1+\mathrm{K}} \rho_{\mathrm{s}} \mathrm{S}(\mathrm{y})
$$

When the reflection factor is constant, the ground resistance of the foot is only proportional to the attenuation coefficient and is proportional. So the thickness of the gravel layer decreases, the attenuation coefficient decreases, grounding resistance is reduced, so the human body suffered step voltage or contact voltage will be reduced.

\section{Appendix C}

When the thickness of the gravel layer is constant, the attenuation coefficient increases as the reflection factor increases. From the equation (B.1) we can see that the grounding resistance and the reflection factor and attenuation coefficient. However, when the reflection factor is increased, the rate of decrease of $1-\mathrm{K} / 1+\mathrm{K}$ is greater than the rate of increase of the attenuation coefficient, so that the grounding resistance is reduced. For example, when $\mathrm{h}=20 \mathrm{~cm}$, when $\mathrm{K}=-0.9$, then $\mathrm{S}(\mathrm{y})=$ $0.8381,1-\mathrm{K} / 1+\mathrm{K}=19$, if $\mathrm{K}$ rises to -0.7 , then $\mathrm{S}(\mathrm{y})=$ $0.8661, \mathrm{~K} / 1+\mathrm{K}=5.6667$. It can be seen, if the reflection factor increases, will result in reduced grounding resistance, so the human body suffered by the step voltage or contact voltage will be reduced.

\section{REFERENCES}

[1] "IEEE Guide for Safety in AC Substation Grounding", ANSI / IEEE Std. 80, 1986.

[2] B. Thapar, V. Gerez, and H. Kejriwal, "Reduction Factor for the Ground Resistance of the Foot in Substation Yards", IEEE Trans. on Power Delivery, Vol.9, No.1, Jan. 1994, pp. $360-368$

[3] Y. Shiau, M.F. Genge, "Step Potential and Body Current Near a Buried Horizontal Bare Conductor in a Two-layer Earth”, IIT Research Institute Technical Report E6357-7, 1977.

[4] W.E. Byerly, "Fourier's Series and Spherical, Cylindrical and Ellipsoidal Harmonics", (book), Ginn and Company, Boston, Mass. 1902.

[5] B. Thapar, V. Gerez, and P. Emmanuel, "Ground Resistance of the Foot in Substation Yards", IEEE Trans. on Power Delivery, Vol.8, No.1, Jan. 1993, pp. 1-6.

[6] E.D. Sunde, "Earth Conduction Effects in Transmission Systems", (book), Dover Publications, Inc. New York, 1968.

[7] J. Zaborszky, "Efficiency of Grounding Grids with Nonuniform Soil", AIEE Transactions, Vol.74, 1955, pp. 1230-1233. 
[8] B. Thapar, V. Gerez, A. Balakrishnan, and D.A. Blank, "Finite Expression and Models for Footing Resistance in Substations", IEEE Trans. on Power Delivery, Vol.7, No.1, Jan. 1992, pp. 219-224.

[9] H.B. Dwight, "Calculation of Resistance to Ground", Electrical Engineering, 1936, pp. 1319-1328.
[10] J. Zaborszky, "Efficiency of Grounding Grids with Nonuniform Soil", AIEE Transactions, Vol.74, 1955, pp. 1230-1233.

[11] "IEEE Standard Procedures for Measurement of Power Frequency Electric and Magnetic Fields from AC Power Lines", ANSI / IEEE Std. 644-1994, 1994. 